\title{
Genitourinary Infections after a Routine Pelvic Exam
}

\author{
Jeffrey D. Tiemstra, MD, Patricia D. Chico, BA, and Emily Pela, MA
}

Purpose: To determine if genitourinary problems are more common in women in the first 1 to 2 months after a routine pelvic examination.

Methods: This was a historical cohort study in 2 family medicine teaching clinics at an urban university. Participants included all women who received a Papanicolaou smear during calendar year 2006. Subjects included all participants for weeks 1 to 7 after their Papanicolaou smear. Controls were all participants in weeks 8 to 52 after their Papanicolaou smear. The independent variable was time after Papanicolaou smear, and dependent variables included frequency of visits for urinary complaints, diagnosed urinary tract infections (UTIs), vaginal complaints, diagnosed vaginitis, and sexually transmitted diseases. Relative frequencies compared using 2-tailed $t$ tests.

Results: UTIs and urinary complaints were significantly more frequent during the first 7 weeks after a Papanicolaou smear compared with weeks 8 to 14, 8 to 48, and 8 to 52. The overall rate of UTIs was 7.33 per 100 person-years; the attributable risk during the first 7 weeks was 0.82 per 100 personyears, or $11 \%$ of observed UTIs. Combined Candidal and bacterial vaginitis was marginally more frequent during the first 7 weeks compared with weeks 8 to 48 and 8 to 52 only. The incidence of sexually transmitted diseases combined was 1.13 per 100 person-years, distributed evenly over the study period unrelated to time.

Conclusions: The risk of UTI is increased in the 7 weeks after a Papanicolaou smear. (J Am Board Fam Med 2011;24:296-303.)

Keywords: Infectious Diseases, Patient Safety, Women's Health

Urinary tract infections (UTIs) are very common among adult women, accounting for more than 6 million physician office visits yearly in the United States. Sexual intercourse has long been recognized as one of the primary risk factors for female UTIs. ${ }^{1}$ Proposed mechanisms for this increased risk include propulsion of bacteria up the urethra into the bladder; microtrauma to the urethra and bladder mucosa, allowing bacterial adhesion; and alteration of normal flora. We postulated that the pelvic examination, including speculum insertion and bimanual examination, may increase the risk of UTIs

This article was externally peer reviewed.

Submitted 6 January 2011; revised 10 January 2011; accepted 19 January 2011.

From Family Medicine Center, University of Illinois at Chicago College of Medicine Chicago.

Funding: none.

Conflict of interest: none declared.

Corresponding author: Jeffrey D. Tiemstra, MD, Family Medicine Center (M/C 397), University Village, 722 West Maxwell Street, Suite 235, Chicago, IL 60607 (E-mail: jtiemstr@uic.edu). by similar mechanisms. A small, retrospective, casecontrol study suggested such an association. ${ }^{2} \mathrm{~A}$ recent study has also demonstrated that symptoms of UTIs, namely dysuria and frequency, are more common in sexually active women in the 4 weeks after a screening pelvic examination. ${ }^{3}$ This study examined a larger cohort of women after a routine pelvic examination for the timing and frequency of genitourinary problems.

\section{Methods}

This was a historical cohort study performed by chart review. Participants were all women who had a Papanicolaou test at either of our 2 clinics during the calendar year 2006. Participants were identified through billing records, and a total of 1582 women were included. Participants were excluded only if they had no follow-up visit at our medical center since the initial Papanicolaou smear. The 2 clinics are university-based family medicine clinics in an urban setting, serving a diverse, large-city popula- 
Table 1. Mean Weekly Urinary Problems after a Pelvic Exam $(n=1582)$

\begin{tabular}{lccccccc}
\hline & $\begin{array}{c}\text { Weeks } 1 \\
\text { to } 7\end{array}$ & $\begin{array}{c}\text { Weeks } 8 \\
\text { to } 14\end{array}$ & $\begin{array}{c}P \text { (vs Weeks } \\
1 \text { to 7) }\end{array}$ & $\begin{array}{c}\text { Weeks } 8 \\
\text { to } 48\end{array}$ & $\begin{array}{c}P \text { (vs Weeks } \\
\text { 1 to 7) }\end{array}$ & $\begin{array}{c}\text { Weeks } 8 \\
\text { to 52 }\end{array}$ & $\begin{array}{c}P(\text { vs Weeks } \\
1 \text { to 7) }\end{array}$ \\
\hline All urinary problems & 4.29 & 2.57 & $.028^{*}$ & 2.37 & $.003^{\dagger}$ & 2.27 & $.002^{\dagger}$ \\
Urinary tract infections & 3.86 & 2.14 & $.016^{*}$ & 2.05 & $.006^{\dagger}$ & 1.98 & $.005^{\dagger}$ \\
\hline
\end{tabular}

${ }^{*} P<.05$

${ }^{\dagger} P<.01$

tion, 30\% of which are members of a university student health plan. Patients were seen by 22 different clinicians during the study period (14 attending physicians, 3 nurse practitioners, and 15 family medicine residents). On average, less than $25 \%$ of Papanicolaou smears in these clinics are performed by residents or students. Subjects were women during the period immediately after their Papanicolaou test, up to 8 weeks after the test; controls were the same population from week 8 to week 52 . The independent variable was time in weeks from the Papanicolaou test (weeks 1 to 7 vs weeks $8-14$, $8-48$, and 8-52). Dependent variables were frequency of visits for urinary problems (UTI, urinary complaint without diagnosis); vaginitis (bacterial, candidal, or undiagnosed); and sexually transmitted diseases (STDs; namely chlamydia, gonorrhea, her- pes, and genital warts). The reason for each visit was recorded by the final diagnosis listed in the chart note. UTI, vaginitis (candidal or bacterial), and specific STDs were classified based on the providers' final diagnosis, whether they based it on history and examination alone or laboratory confirmation. If no final diagnosis was made but the chief complaint was urinary or vaginal symptoms, then the visit was classified as a urinary or vaginal problem. Analysis was performed using 2-tailed $t$ tests.

\section{Results}

\section{Urinary Events}

UTIs occurred at a rate of 12.7 per 100 personyears during the first 7 weeks after a Papanicolaou

Figure 1. Weekly visits for urinary problems after a pelvic examination.

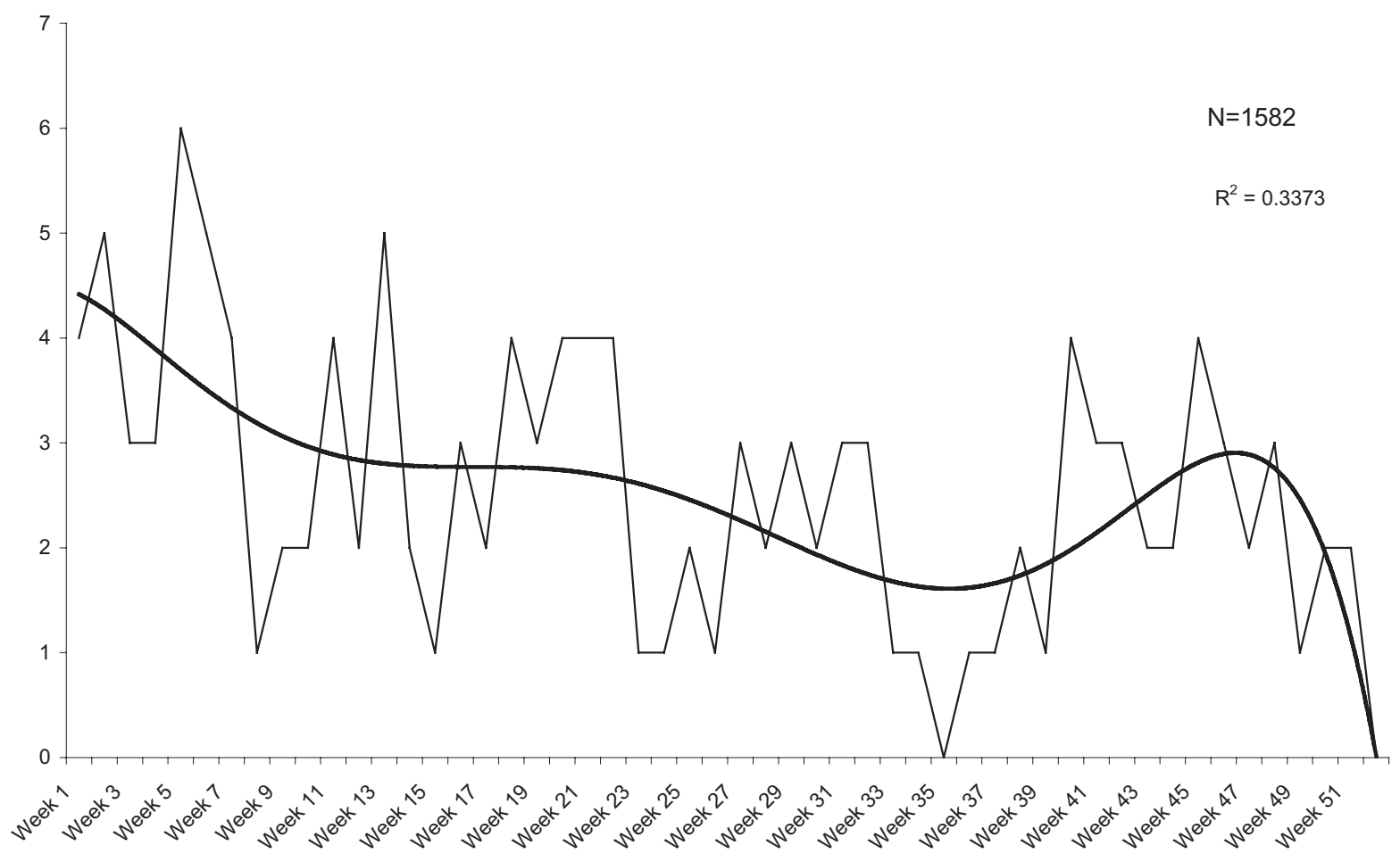


smear compared with 6.51 per 100 person-years for weeks 8 to $52(P<.01)$. Table 1 shows mean weekly rates for the first 7 weeks compared with weeks 8 to 14, 8 to 48, and 8 to 52; Figures 1 and 2 illustrate the incidence of urinary problems and UTIs, respectively. Comparison was done using weeks 8 to 48 as the control because of the drop-off in UTIs observed during weeks 49 to 52 . Comparison was also done using weeks 8 to 14 as the control to minimize any gradual patient drop-out over time. In all comparisons a significantly higher incidence in weeks 1 to 7 was observed.

\section{Vaginal Events}

Figures 3 and 4 illustrate the incidence of all vaginal problems and diagnosed vaginitis, respectively, and Table 2 shows the mean weekly rates. Though the pattern resembles that of urinary events, only diagnosed vaginitis compared with weeks 8 to 48 and 8 to 52 reached significance at the $P<.05$ level.

\section{STDs}

Figure 5 illustrates the weekly incidence of STDs. The overall incidence was 1.13 per 100 personyears, distributed evenly over the time period. The
5 STDs in week 1 were all cases of asymptomatic chlamydia identified on routine screening performed in conjunction with the Papanicolaou test, and by chart review none of those patients had genitourinary symptoms recorded at the time of their visit $(0.3 \%$ asymptomatic carrier rate).

\section{Discussion}

Considering alternate explanations for the observed increase in urinary problems after the pelvic examination, we must first consider whether patients come in for a Papanicolaou test because they are having symptoms that they may or may not report. Given the observed increased incidence in the first 2 weeks, this may be a possibility. However, the fact that the observed increase persisted through week 7 argues against infections already present at the initial visit for the Papanicolaou smear, suggesting an effect of events around the time of the examination. Furthermore, patients who have genitourinary symptoms at the time of their Papanicolaou smear are likely to report these symptoms either directly or during the clinician's review of these systems, in which case they will likely be treated and will not need to return for

Figure 2. Weekly visits for diagnosed utis after a pelvic examination.

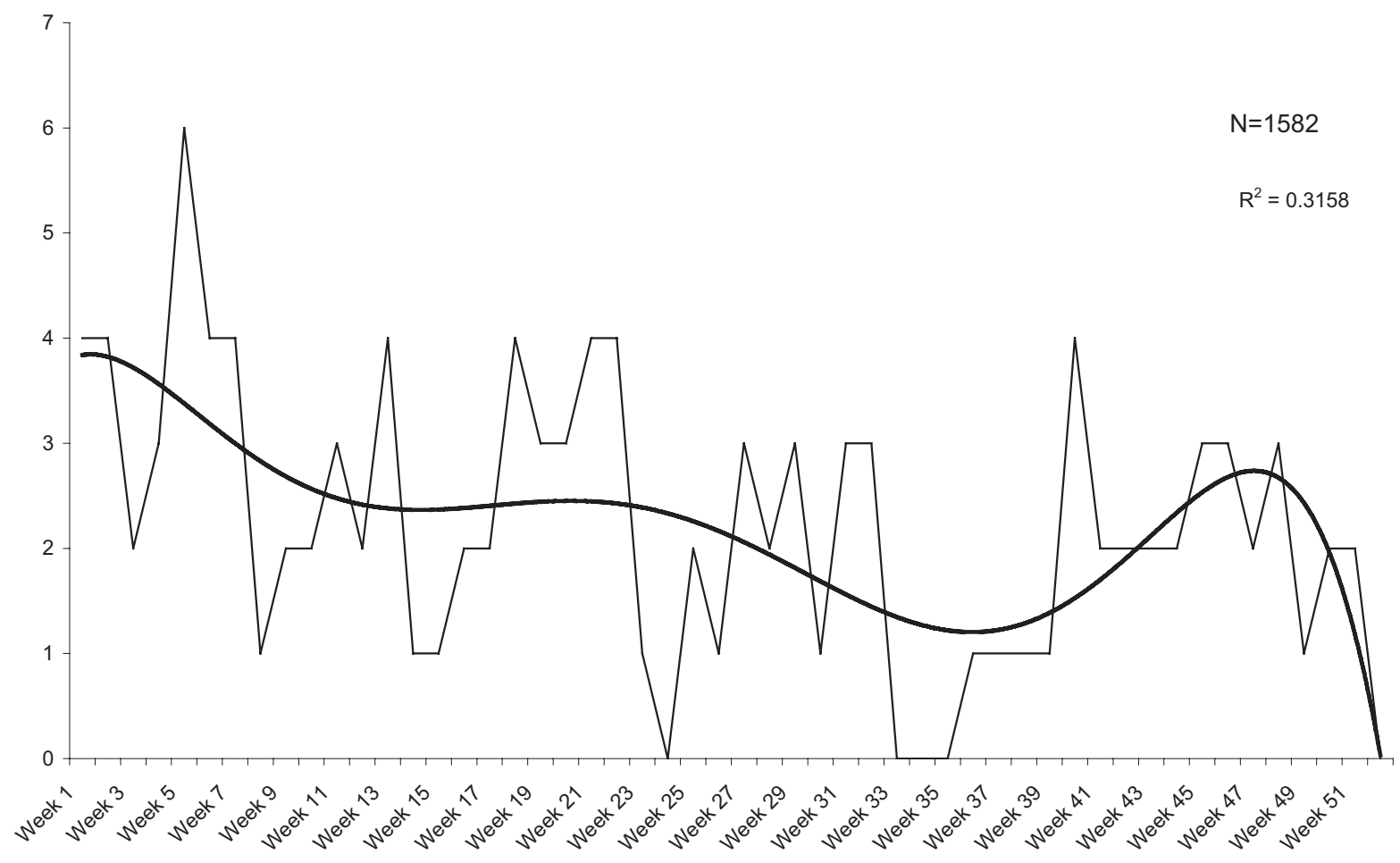


Figure 3. Weekly visits for vaginal problems after a pelvic examination.

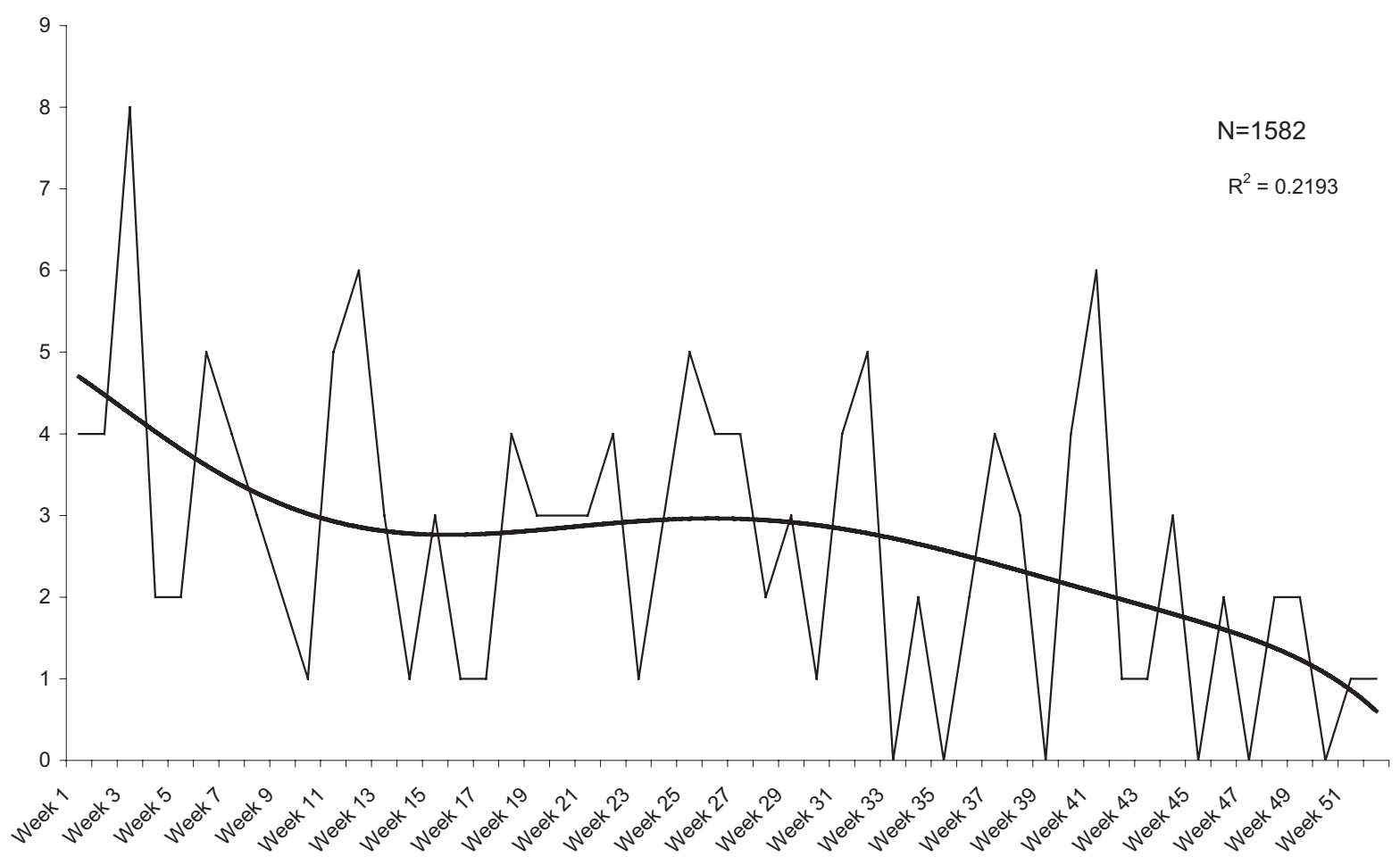

further treatment. In fact, treatment with antibiotics for a UTI at the time of the Papanicolaou smear examination triggering a UTI. Only patients who had symptoms that they did not report at the time would likely reduce the possibility of the pelvic of the Papanicolaou smear (and thus that would not

Figure 4. Weekly visits for candidiasis/bacterial vaginosis after a pelvic examination.

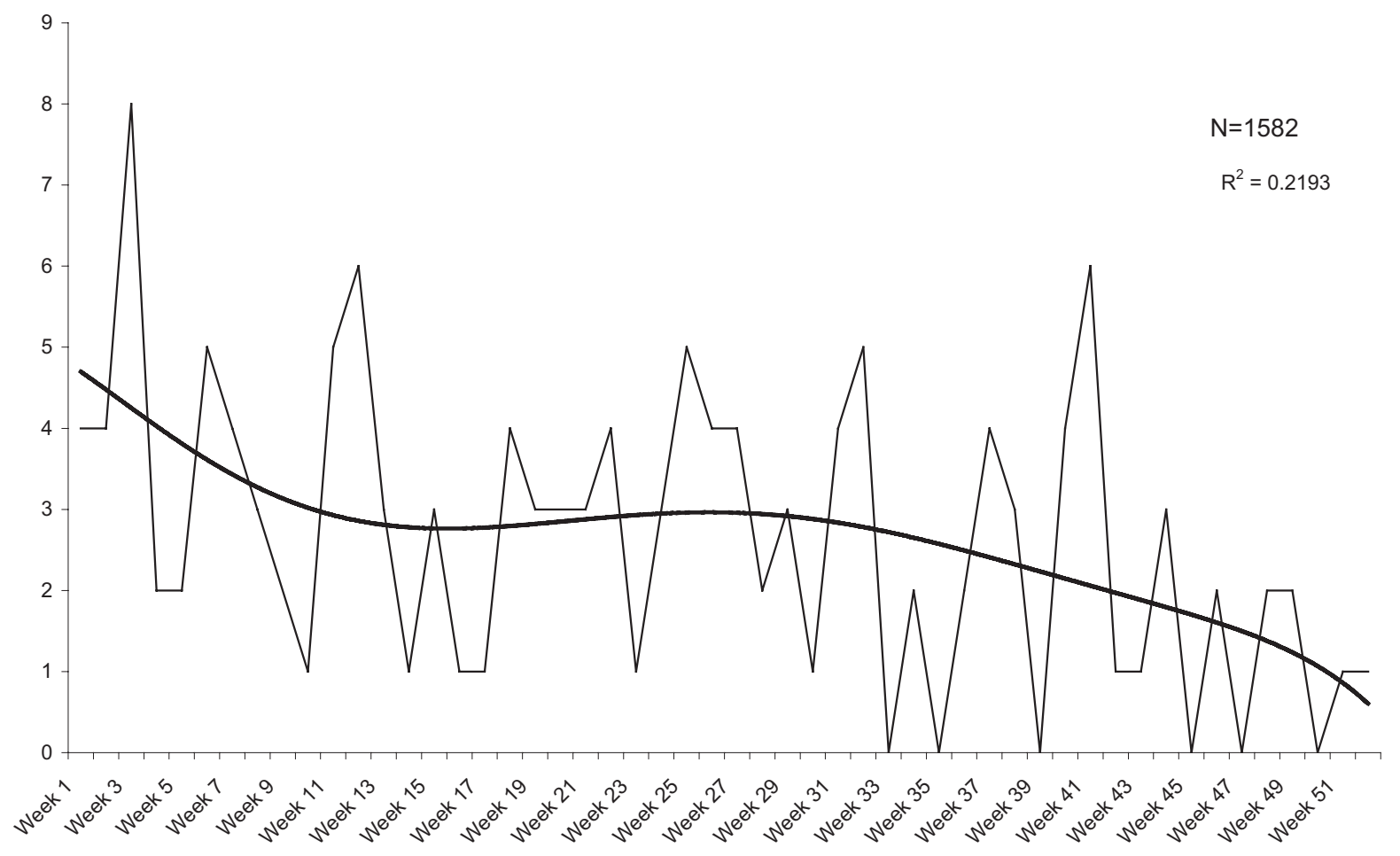


Table 2. Mean Weekly Vaginal Problems after a Pelvic Exam $(n=1582)$

\begin{tabular}{lcccccccc}
\hline & $\begin{array}{c}\text { Weeks } 1 \\
\text { to } 7\end{array}$ & $\begin{array}{c}\text { Weeks } 8 \\
\text { to } 14\end{array}$ & $\begin{array}{c}P \text { (vs Weeks } \\
1 \text { to 7) }\end{array}$ & $\begin{array}{c}\text { Weeks } 8 \\
\text { to 48 }\end{array}$ & $\begin{array}{c}P \text { (vs Weeks } \\
\text { 1 to 7) }\end{array}$ & $\begin{array}{c}\text { Weeks } 8 \\
\text { to 52 }\end{array}$ & $\begin{array}{c}P(\text { vs Weeks } \\
1 \text { to 7) }\end{array}$ \\
\hline All vaginal problems & 4.14 & 3.00 & 0.301 & 2.56 & .093 & 2.42 & .071 \\
Candidiasis/bacterial vaginosis & 2.86 & 2.29 & .545 & 1.85 & $.030^{*}$ & 1.76 & $.019^{*}$ \\
Candidiasis & 1.71 & 1.57 & .858 & 1.12 & .106 & 1.07 & .077 \\
Bacterial vaginosis & 1.14 & 0.71 & .207 & 0.73 & .185 & 0.69 & .145 \\
\hline
\end{tabular}

${ }^{*} P<.05$

be in the medical record) would be likely to return for their symptoms in the near future, and the number of these patients would likely be very small. Therefore we did not attempt to exclude patients who reported symptoms at the time of their Papanicolaou smear. Doing so would have excluded patients who would have likely been successfully treated and would not have needed to return, but it would not have excluded patients who may have had mild symptoms they did not report.

Another alternate explanation would be that some patients come in for a Papanicolaou test in anticipation of increased or renewed sexual activity, which may also increase their risk of UTI in the near future. This study cannot rule out this possibility, although the lack of an observed increase in
STDs in this population in the time frame immediately after Papanicolaou smears argues against this as a major factor. Another question related to sexual activity is whether intercourse is a necessary cofactor for the pelvic examination to trigger UTI; ie, were patients who were not sexually active at increased risk for UTI after the pelvic examination? Because this was a chart review study we did not feel we could reliably obtain information about the frequency and patterns of intercourse among participants. Whether intercourse is a necessary cofactor would be important when considering preventive strategies, but it is not central to the question of the pelvic examination itself as a risk factor.

Another concern in this study is patient dropout. We deliberately chose a time frame 3 years in

Figure 5. Weekly visits for sexually transmitted diseases after a pelvic examination.

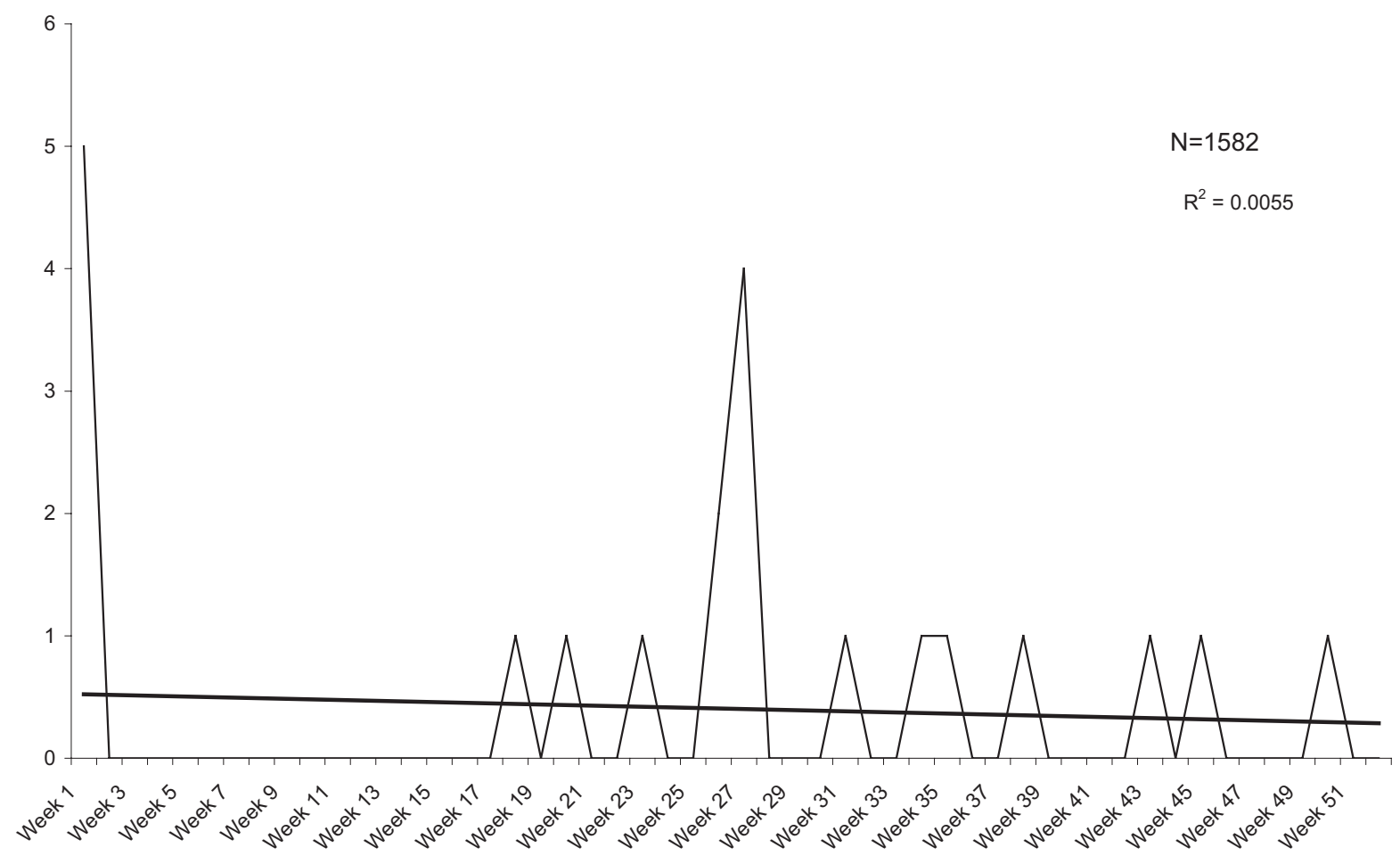


Figure 6. Weekly visits for diagnosed urinary tract infections in weeks 8 to 52 after a pelvic examination.

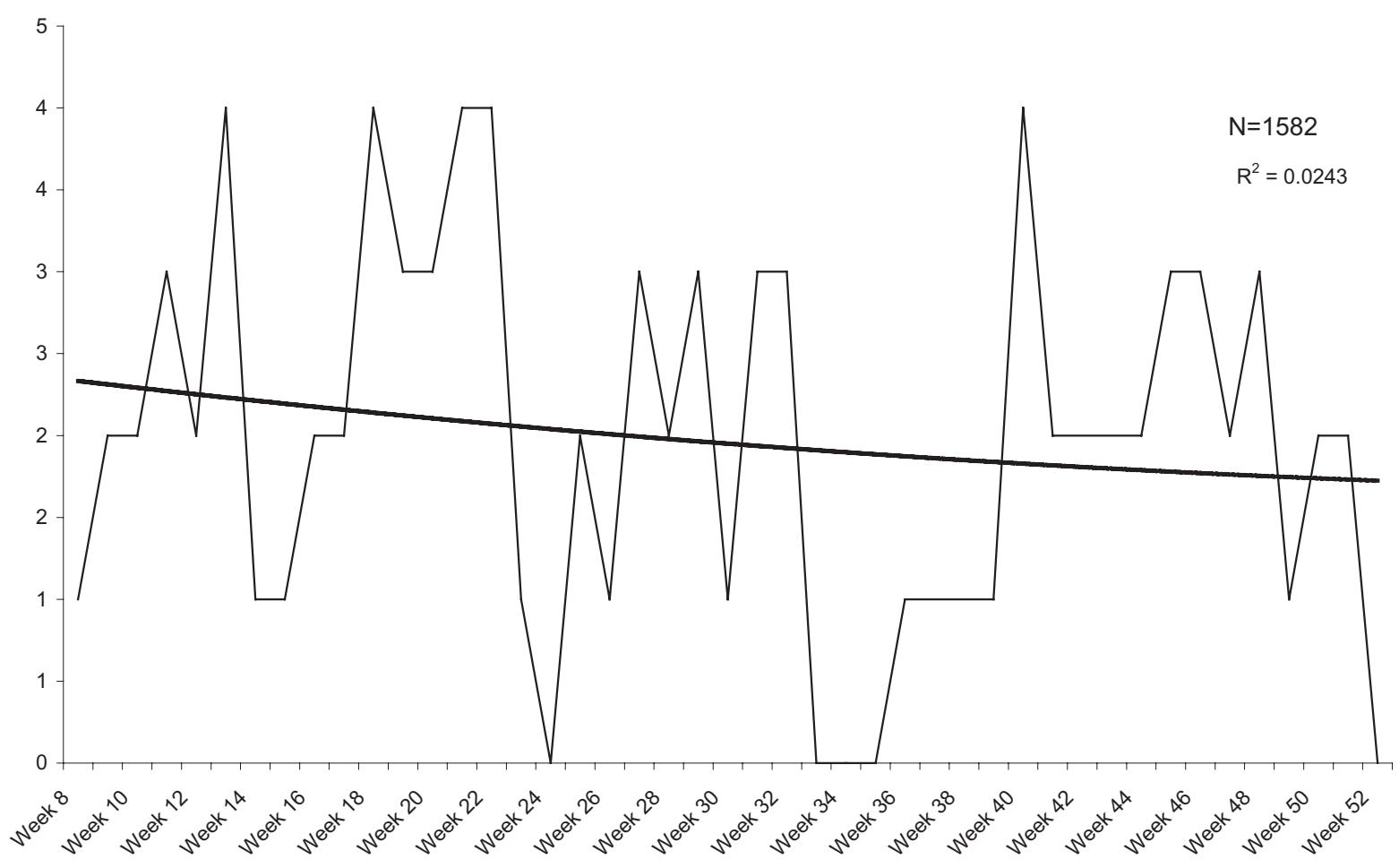

the past so that we could document that patients who go to the doctor infrequently could be included with some confidence that they were continuing their care in these clinics. This does not rule out the possibility that some patients sought care elsewhere during the study period. However, the distributions of all events and infections do not follow linear declines, but instead demonstrate significant declines in the first 7 weeks followed by plateaus for the remainder of the follow-up period. Figures 6 and 7 show UTIs and vaginitis during weeks 8 to 52 and illustrate plateaus with very minimal decline. To reduce the effect of any gradual decline over time, we compared infection and event rates for weeks 1 to 7 with weeks 8 to 14 and confirmed a statistically higher rate of UTIs in the first 7 weeks. Because both urinary and vaginal events and infections did drop off during the last 4 weeks, we also performed analysis excluding this data, comparing weeks 1 to 7 with weeks 8 to 48 , and found similar results.

Our results also raise the question of whether an effect of the pelvic examination could be responsible for UTIs occurring up to 6 to 7 weeks later. The week-to-week variations in the UTI rate in our study do not allow a precise characterization of the timing after the pelvic examination, but do suggest increased risk for up to 6 to 7 weeks. Comparing microtrauma and inoculation of bacteria from a pelvic examination with that occurring with intercourse, sexually active patients usually have intercourse on a recurring basis, so there is no clear answer from the literature on the timing of a UTI after a single episode of intercourse. An understanding of the pathophysiology of UTIs is still evolving, but recent study has demonstrated that inoculation of the bladder with Escherichia coli leads to initial superficial colonization of the epithelium and bacteruria that is often cleared by the host. However, persistent colonization of the bladder epithelium can remain for weeks in the absence of detectable bacteruria and can trigger UTI at a much later time. ${ }^{4}$ This is being studied in the context of patients with recurrent UTIs, but can also explain isolated UTIs that occur weeks after an initial bacterial inoculum. Based on this research it seems reasonable that any effect produced by the pelvic examination may persist for several weeks before dropping off to a baseline rate.

Because this study was performed in a teaching practice, we cannot rule out an exaggerated effect of the pelvic examination by less skilled examiners. However, less than $25 \%$ of Papanicolaou smears in the clinic are performed by residents, 
Figure 7. Weekly visits for diagnosed vaginitis in weeks 8 to 52 after a pelvic examination.

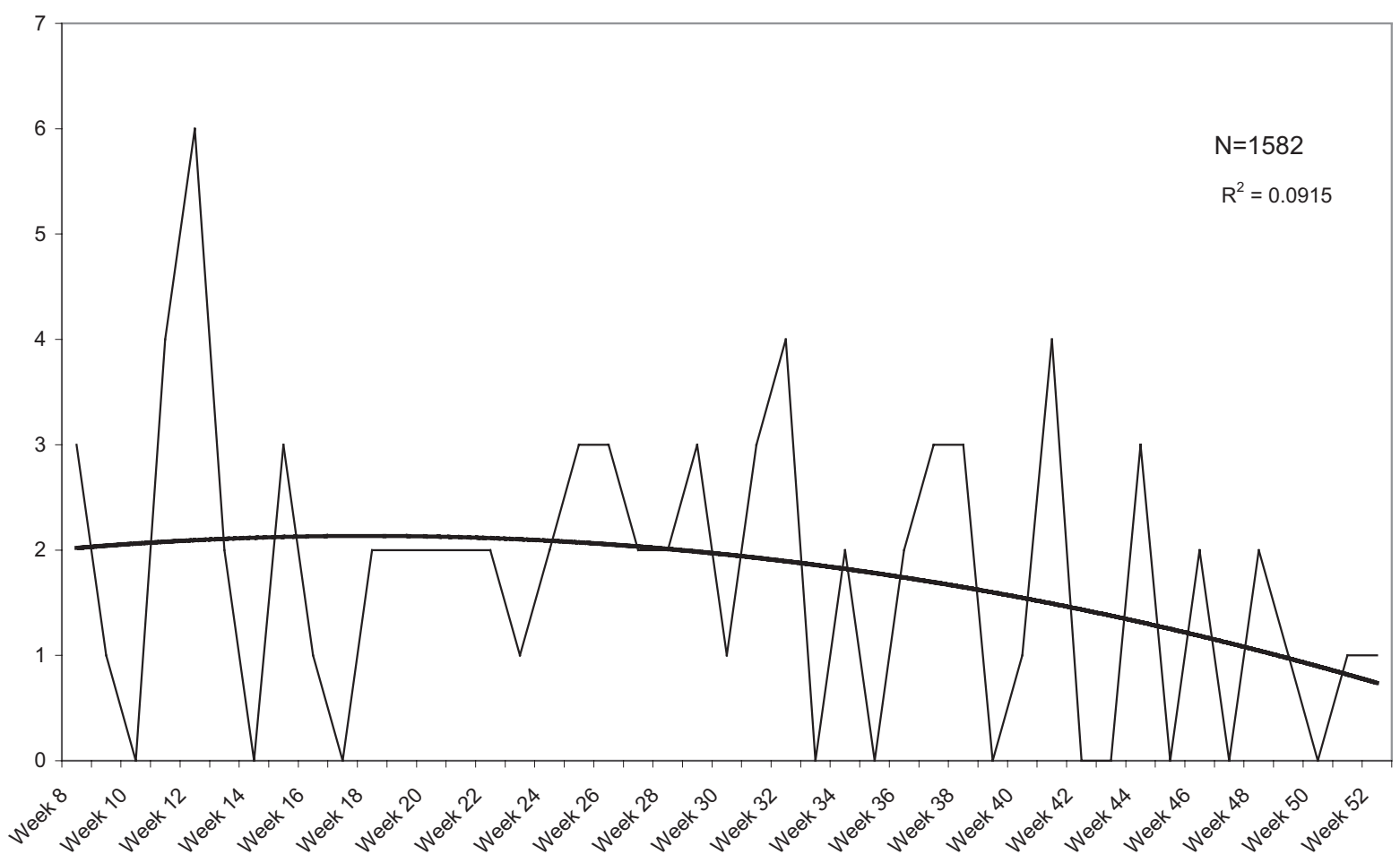

with the majority being done by attending physicians or nurse practitioners. We also did not attempt to determine whether patients had a speculum examination only or a bimanual examination as well. It is the general practice of the majority of our clinicians to routinely perform bimanual examinations, so the number of patients who did not have a bimanual examination would be small. Because it is likely that both the speculum and bimanual exams can produce some degree of microtrauma and contribute to a possible effect, including patients who had only a speculum examination would likely diminish any observed effect of the pelvic examination. Finally, although individual clinician factors such as technique may also affect risk, by including 22 different clinicians, any idiosyncratic variations that might have excessively increased risk should have been minimized.

The marginally significant increase in diagnosed vaginitis is also of interest. The possibility that the use of a speculum and lubricant may alter normal floral and trigger vaginitis in a few patients seems reasonable, although an association is certainly not confirmed by this study.

If we assume that the observed increase in UTIs is in fact causally related to the pelvic examination, then we can estimate the relative risk posed by the pelvic examination. The overall risk of UTI in this population was 7.33 events per 100 person-years (weeks $1-52$ ), and the risk of UTI among controls was 6.51 events per 100 person-years (weeks 8-52). This yields an attributable risk of 0.82 events per 100 person-years, or $11 \%$ of all observed UTIs. On an individual patient level this represents a very small risk of $0.83 \%$ for an annual pelvic examination. However, at a population level, with 50 million Papanicolaou smears performed annually in the United States, this represents 415,000 UTIs annually, or $6.9 \%$ of all UTIs by this estimate.

\section{Conclusions}

The Papanicolaou smear has played a dramatic role in reducing the mortality and morbidity from cervical cancer, and we would not want to deter patients from obtaining this potentially life-saving test at recommended intervals. However, that concern should not prevent us from exploring the potential for safer methods of screening, and there are several possibilities. First, adherence to new guidelines for less frequent screening in women with a history of normal Papanicolaou smears would reduce the number of unnecessary exams in low-risk 
women. Second, although our study does not delineate the contribution of the bimanual examination to the increased risk of UTI, it seems reasonable to assume that it contributes, at least in part, to urinary tract irritation. Because there is no data supporting the bimanual examination as an effective screening tool, consideration should be given to eliminating it from the routine pelvic examination during Papanicolaou smear screening. Finally, there is substantial new research about the effectiveness of patient self-sampling for cervical cancer screening. The accuracy of self-collected Papanicolaou smears is unproven, with variations in accuracy depending on the technique employed. ${ }^{5,6}$ However, self-sampling for the human papillomavirus is emerging as an accurate and effective technique for cervical cancer screening through a variety of methods. ${ }^{7-10}$ Although self-sampling research has been more focused on developing countries where access to providers is limited, self-sampling techniques that avoid speculum insertion deserve further consideration for all populations as alternatives that, in addition to being potentially less traumatic, may also increase screening rates.

\section{References}

1. Griebling TL. Urinary tract infections in women. In: Litwin MS, Saigal CS, eds. Urologic diseases in America. NIH publication no. 04-5512. US Department of Health and Human Services, Public Health Service, National Institutes of Health, National Institute of Diabetes and Digestive and Kidney Diseases. Washington, DC: US Government Publishing Office; 2004:153-183.
2. Tiemstra, JD, Sinacore JM. The relationship between urinary tract infection and a recent pelvic examination in women. Arch Fam Med 1996;5:357-60.

3. Tiemstra JD, Pela E. Urinary symptoms in women following a routine pelvic exam. J Am Board Fam Med 2011;24:xxx.

4. Mulvey MA, Schilling JD, Hultgren SJ. Establishment of a persistent Escherichia coli reservoir during the acute phase of a bladder infection. Infect Immun 2001;69(7):4572-9.

5. Pengsaa P, Sriampom S, Kritpetcharat O, et al. A comparison of cytology with Pap smears taken by a gynecologist and with a self-sampling device. Asian Pac J Cancer Prev 2003;4:99-102.

6. Budge M, Halford J, Haran M, et al. Comparison of a self-administered tampon Thinprep test with conventional pap smears for cervical cytology. Aust N Z J Obstet Gynaecol 2005;45:215-9.

7. Stewart DE, Gagliardi A, Johnston M, et al. Selfcollected samples for testing of oncogenic human papillomavirus: a systematic review. J Obstet Gynaecol Can 2007;29:817-28.

8. Bhatla N, Dar L, Patro AR, et al. Can human papillomavirus DNA testing of self-collected vaginal samples compare with physician-collected cervical samples and cytology for cervical cancer screening in developing countries? Cancer Epidemiol 2009;33: 446-50.

9. Eijsink JJ, Yang N, Lendvai A, et al. Detection of cervical neoplasia by DNA methylation analysis in cervico-vaginal lavages, a feasibility study. Gynecol Oncol 2011;120:280-3.

10. Richman AR, Brewer NT, Liebman AK, Rinas AC, Smith JS. Optimising human papillomavirus selftesting for high risk women. Sex Trasm Infect 2011; $87: 118-22$. 